\begin{tabular}{|l|l|}
\hline OP0132 & ALLERGIC ASTHMA INDUCES THE ACCUMULATION \\
OF SYNOVIAL RESIDENT EOSINOPHILS, TRIGGERING \\
THE RESOLUTION OF INFLAMMATORY ARTHRITIS
\end{tabular}

M. Liu ${ }^{1}$, D. Andreev ${ }^{1}$, K. Kachler ${ }^{1}$, J. Koelle ${ }^{2}$, S. Rauber ${ }^{1}$, A. Ramming ${ }^{1}$, S. Finotto ${ }^{2}$, G. Schett ${ }^{1}$, A. Bozec ${ }^{1} .{ }^{1}$ University Hospital Erlangen and Friedrich Alexander University of Erlangen-Nürnberg (FAU), Department of Medicine 3, Rheumatology and Immunology, Erlangen, Germany; ${ }^{2}$ University Hospital Erlangen and FAU, Department of Molecular Pneumology, Erlangen, Germany

Background: Rheumatoid arthritis (RA) is a chronic inflammatory disorder, involving synovial joints, which affects approximately 1 percent of the world population[1]. Our former work demonstrated that the Th2-eosinophil pathway is a strong anti-inflammatory mediator of inflammatory arthritis[2]. Allergic asthma is an inflammatory disease of the airway, triggered by type 2 immune response. Hitherto, clinical observations on the impact of asthma on RA showed controversial results. Herein, we investigated the action of allergic asthma on inflammatory arthritis.

Objectives: We aimed to delineate the molecular and cellular responses induced by allergic asthma on inflammatory arthritis, particularly depicting the role of eosinophil subsets in arthritic synovium.

Methods: Allergic asthma was induced in wild type and genetically modified mice by ovalbumin (OVA) treatment. After the initiation of allergic asthma, $\mathrm{K} /$ $\mathrm{BxN}$ serum was transferred into the asthmatic mice or control mice to trigger serum induced arthritis (SIA). Then, arthritis severity, circulating cytokines and the cytology of lung and synovium were analyzed. Eosinophil subsets were studied by flow cytometry, single cell RNA sequencing analysis, and were isolated and transferred into the synovial cavity of eosinophil deficient arthritic mice. Clinical data of patients with both RA and asthma were collected and checked for the relapse of RA after asthma treatment with anti-interleukin (IL)-5 antibody.

Results: Mice induced with allergic asthma exhibited a rapid resolution of SIA. The OVA-triggered resolution disappeared in eosinophil deficient mice ( $\triangle$ dblGATA), and was partially blocked by IL-5 neutralization. We could detect that IL-5 was mainly produced by type 2 innate lymphoid cell (ILC2) in the lung. Allergic asthma exclusively induced the proliferation $\left(\mathrm{Ki} 7^{+}\right)$and accumulation of synovial resident eosinophils ( $\mathrm{rEos}$, Siglec- $\mathrm{F}^{\text {int }}$ ), which switched classical macrophages into alternatively activated macrophages. Synovial induced eosinophils (iEos, Siglec- $\mathrm{F}^{\text {high }}$ ) appeared only in the acute phase of SIA. Single cell RNA sequencing analysis showed that $\mathrm{rEos}$ played an anti-inflammatory role, while iEos had pro-inflammatory properties in arthritis. The roles of rEos and iEos in arthritis were confirmed by transferring $\mathrm{rEos} / \mathrm{iEos}$ into the synovial cavity of arthritic mice. Patiens with both RA and asthma showed a remission relapse of RA after using humanized monoclonal IL-5 antibody for treating sever eosinophilic asthma.

Conclusion: Allergic asthma induced an IL-5 mediated proliferation and accumulation of synovial rEos. The latter triggered the resolution of inflammatory arthritis. In human, eosinophils induced by asthma were essential for the sustaining of RA remission.

References:

[1] Myasoedova, E., et al., Is the incidence of rheumatoid arthritis rising? results from Olmsted County, Minnesota, 1955-2007. Arthritis Rheum, 2010. 62(6): p. 1576-82.

[2] Chen, Z., et al., Th2 and eosinophil responses suppress inflammatory arthritis. Nat Commun, 2016. 7: p. 11596

Acknowledgments: Mengdan Liu and Darja Andreev contributed equally to this study

Disclosure of Interests: Mengdan Liu: None declared, Darja Andreev: None declared, Katerina Kachler: None declared, Julia Koelle: None declared, Simon Rauber: None declared, Andreas Ramming Grant/research support from: Pfizer, Novartis, Consultant of: Boehringer Ingelheim, Novartis, Gilead, Pfizer, Speakers bureau: Boehringer Ingelheim, Roche, Janssen, Susetta Finotto: None declared, Georg Schett Speakers bureau: AbbVie, BMS, Celgene, Janssen, Eli Lilly, Novartis, Roche and UCB, Aline Bozec: None declared DOI: 10.1136/annrheumdis-2020-eular.4479

\section{OP0133 \\ PRECLINICAL EFFICACY OF R835, A NOVEL IRAK1/4 DUAL INHIBITOR, IN RODENT MODELS OF JOINT INFLAMMATION}

C. Lamagna ${ }^{1}$, M. Chan ${ }^{1}$, E. Tai ${ }^{1}$, S. Siu ${ }^{1}$, R. Frances ${ }^{1}$, S. Yi ${ }^{1}$, C. Young ${ }^{1}$ V. Markovtsov ${ }^{1}$, Y. Chen ${ }^{1}$, L. Chou', G. Park', E. Masuda', V. Taylor ${ }^{1} .{ }^{1}$ Rigel Pharmaceuticals, South San Francisco, United States of America

Background: Interleukin receptor associated kinases (IRAK) 1 and 4 are kinases involved in Toll-Like Receptor (TLR) and Interleukin-1 Receptor (IL-1R) signaling pathways, which regulate innate immunity and inflammation. Dysregulation of IRAK $1 / 4$ signaling can lead to a variety of inflammatory conditions including rheumatoid and gouty arthritis. As a result, IRAK $1 / 4$ are promising therapeutic targets for rheumatic diseases (1). We have identified a potent and selective IRAK1/4 inhibitor, R835, that substantially suppressed the elevation of LPS (TLR4 agonist)-induced serum cytokines in healthy human volunteers in a recently completed phase 1 study.

Objectives: The aim of our study was to investigate the effect of IRAK1/4 selective inhibition as a potential therapeutic approach for rheumatological diseases We evaluated the inhibition by our clinical candidate, R835, on TLR-, IL-1R- and NLRP3 inflammasome-induced cytokine production, as well as in preclinical models of arthritis.

Methods: The effect of R835 on TLR- or IL-1R-induced cytokine production was evaluated in vitro using THP-1, human primary endothelial cells and human primary dendritic cells. The activity of R835 on the NLRP3 inflammasome was also tested in vitro using THP-1 cells. The pharmacokinetic-pharmacodynamic relationship of R835 was evaluated in a mouse model of IL-1b-induced cytokine release. Mice were pre-treated orally with vehicle or R835 prior to challenge; serum cytokine and plasma compound levels were determined. The efficacy of IRAK1/4 inhibition by R835 in rodent models of joint inflammation was evaluated in a mouse model monosodium (MSU)-induced peritonitis, in rat model of MSU-induced gouty arthritis and in a rat model of collagen-induced arthritis (CIA).

Results: In human cells, R835 blocked proinflammatory cytokine production in response to TLR, IL-1R and NLRP3 inflammasome activation. In mice, R835 dose-dependently decreased serum cytokines in response to administration of IL-1b. Mice pre-treated with R835 demonstrated dose-dependent reductions in MSU crystal-induced serum and peritoneal cytokine levels, as well as neutrophil influx in the peritoneal cavity. Prophylactic and therapeutic treatment with R835 also resulted in significant inhibition of MSU crystal-induced knee edema and pain in a rat model of human gouty arthritis. In the rat model of CIA, R835 blocked both onset and progression of disease, by reducing inflammation, cartilage degeneration and synovial inflammation.

Conclusion: R835 is a promising clinical candidate for the treatment of a range of cytokine-driven rheumatological diseases. R835 has proven to have desirable pharmacokinetic properties, was well tolerated and suppressed LPS-induced serum cytokines in healthy volunteers in a recent phase 1 study.

References:

[1] Bahia M S, Kaur M, Silakari P, Silakari O. Interleukin-1 receptor associated kinase inhibitors: Potential therapeutic agents for inflammatory- and immune-related disorders. Cellular Signalling 27 (2015) 1039-1055.

Disclosure of Interests: Chrystelle Lamagna Shareholder of: Rigel Pharmaceuticals, Employee of: Rigel Pharmaceuticals, Meagan Chan Shareholder of: Rigel Pharmaceuticals, Employee of: Rigel Pharmaceuticals, Ernest Tai Shareholder of: Rigel Pharmaceuticals, Employee of: Rigel Pharmaceuticals, Stacey Siu Shareholder of: Rigel Pharmaceuticals, Employee of: Rigel Pharmaceuticals, Roy Frances Shareholder of: Rigel Pharmaceuticals, Employee of: Rigel Pharmaceuticals, Sothy Yi Shareholder of: Rigel Pharmaceuticals, Employee of: Rigel Pharmaceuticals, Chi Young Shareholder of: Rigel Pharmaceuticals, Employee of: Rigel Pharmaceuticals, Vadim Markovtsov Shareholder of: Rigel Pharmaceuticals, Employee of: Rigel Pharmaceuticals, Yan Chen Shareholder of: Rige Pharmaceuticals, Employee of: Rigel Pharmaceuticals, Lu Chou Shareholder of: Rigel Pharmaceuticals, Employee of: Rigel Pharmaceuticals, Gary Park Shareholder of: Rigel Pharmaceuticals, Employee of: Rigel Pharmaceuticals, Esteban Masuda Shareholder of: Rigel Pharmaceuticals, Employee of: Rigel Pharmaceuticals, Vanessa Taylor Shareholder of: Rigel Pharmaceuticals, Employee of: Rigel Pharmaceuticals

DOI: 10.1136/annrheumdis-2020-eular. 1901

OP0134

MACROPHAGES SKEWED BY GM-CSF PRODUCE YKL-40, INSTIGATING ANGIOGENESIS IN GIANT CELL ARTERITIS

Y. Van Sleen ${ }^{1}$, W. F. Jiemy ${ }^{1}$, S. A. Pringle ${ }^{1}$, W. Abdulahad ${ }^{1}$, K. Van der Geest ${ }^{1}$, M. Sandovici ${ }^{1}$, P. Heeringa ${ }^{1}$, E. Brouwer ${ }^{1}$, A. Boots' ${ }^{1}{ }^{1}$ University Medical Center Groningen, Groningen, Netherlands

Background: Giant cell arteritis (GCA) is an inflammatory disease affecting the medium- and large-sized arteries. The pathology of GCA is characterized by an infiltrate of mainly CD4+ T-cells and macrophages. These macrophages release a wide range of inflammatory, tissue destructive and proangiogenic proteins, including YKL-40. Previously, we demonstrated that macrophage populations in the vessel wall of GCA patients are heterogeneous; one such macrophage subset highly expressed CD206 and MMP-9, and was located in or near the media layer. Cancer studies have implicated YKL-40 production by tumor-associated macrophages in various inflammatory and tissue remodeling processes, 
including angiogenesis. Less is known about the role of YKL-40 in inflammatory diseases such as GCA.

Objectives: Our objective was to investigate the cellular source and the pro-angiogenic function of YKL-40 in GCA patients.

Methods: For this study we performed immunohistochemistry $(\mathrm{IHC})$ and cell culture experiments. IHC for YKL-40 and CD206 was performed on GCA positive temporal artery biopsies (TABs; $n=12$ ) and GCA positive aortas $(n=10)$ of treatment-naive patients. Expression of YKL-40 by macrophages was confirmed by double staining with macrophage transcription factor PU.1. Additionally, the TABs were stained for IL-13Ra2, recently described as the receptor for YKL-40. The effect of skewing signals on YKL-40 production was assessed by cell culture of monocyte-derived macrophages of GCA patients with either M-CSF or GM-CSF $(n=8)$. Subsequently, the supernatant was assayed by ELISA. Finally, the angiogenic potential of YKL-40 was investigated by tube formation experiments using human microvascular endothelial cells (HMVECs).

Results: YKL-40 is produced by a distinct subset of macrophages in GCA TABs and aortas, usually located in or near the media (Figure 1 shows representative stainings in consecutive slides of a GCA TAB). We here show YKL-40 to be expressed by CD206+/MMP-9+ macrophages in all GCA TABs and aortas. In vitro, macrophages were found to produce YKL-40 (Figure 2 shows an increasing YKL-40 production during the maturation of monocytes towards macrophages over 8 days of culture). GM-CSF stimulation, which is known to upregulate CD206 expression in macrophages, gave rise to higher YKL-40 production by GCA macrophages, when compared to M-CSF stimulated macrophages from GCA patients $(\mathrm{p}=0.038)$. In addition, YKL-40 stimulation of HMVECs induced more tube formation compared to unstimulated HMVECs. Finally, we showed, by IHC, abundant expression of the YKL-40 receptor IL-13Ra2 in TABs of GCA patients.

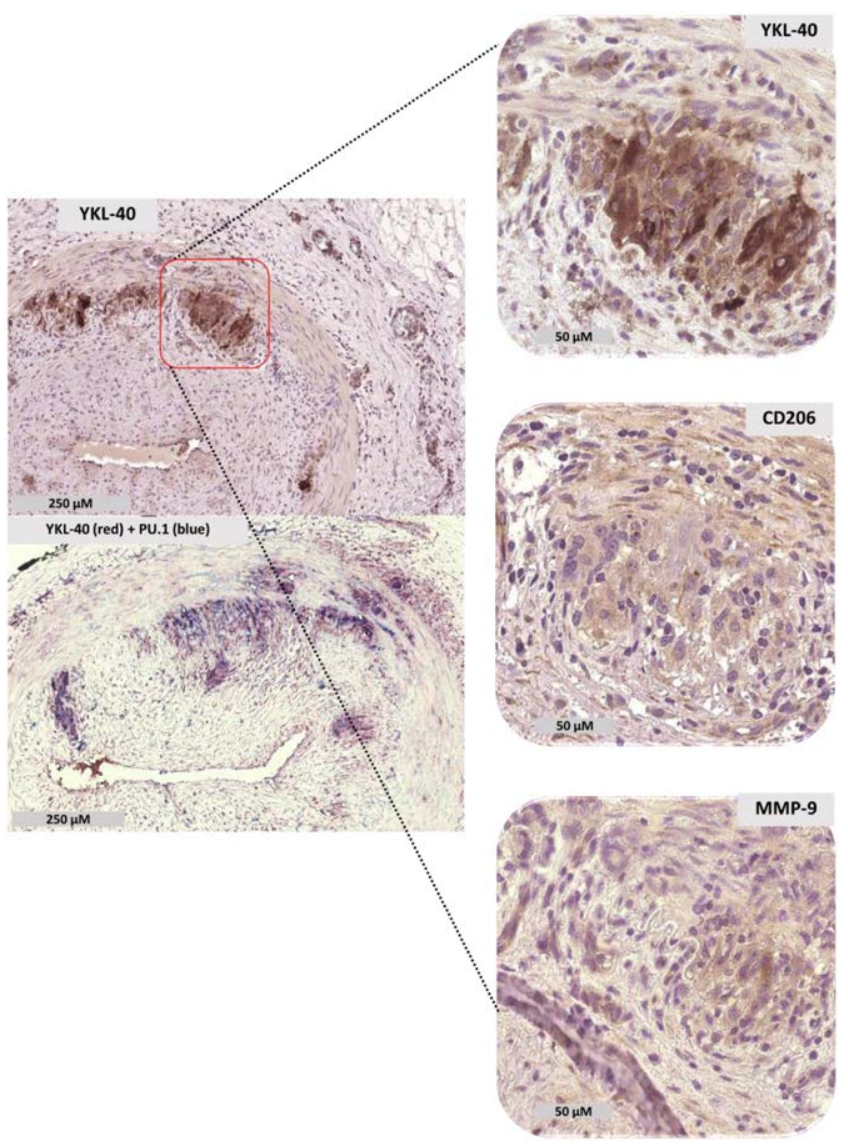

Figure 1

Conclusion: Taken together, we show here that a distinct subset of macrophages, skewed by GM-CSF and highly positive for CD206, is responsible for the production of YKL-40 in GCA. The results are in line with previous reports demonstrating that CD206 expression distinguishes YKL-40 positive macrophages from YKL-40 negative macrophages (1). Thus, YKL-40 production by CD206+ macrophages may be involved in angiogenesis in GCA tissues, a process important for the continuation of the inflammatory process.

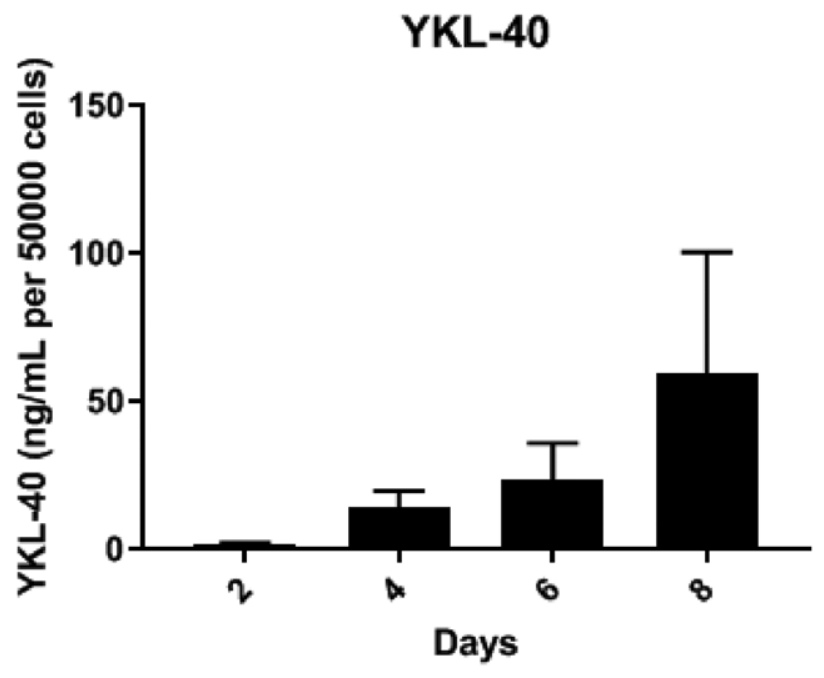

Figure 2

References:

[1] Bonneh-Barkay, D. et al., 2012. Astrocyte and macrophage regulation of YKL40 expression and cellular response in neuroinflammation. Brain Path. 22: 530-546

Disclosure of Interests: Yannick van Sleen: None declared, William Febry Jiemy: None declared, Sarah A. Pringle: None declared, Wayel Abdulahad: None declared, Kornelis van der Geest Speakers bureau: Roche (2019), Maria Sandovici: None declared, Peter Heeringa: None declared, Elisabeth Brouwer Consultant of: Roche (consultancy fee 2017 and 2018 paid to the UMCG), Speakers bureau: Roche (2017 and 2018 paid to the UMCG), Annemieke Boots Consultant of: Grünenthal Gmbh until 2017

DOI: 10.1136/annrheumdis-2020-eular.4455

\section{Connective tissue diseases - genomics, proteomics and pathogenesis}

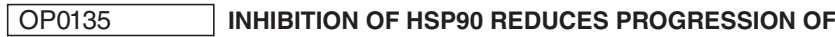 DERMAL FIBROSIS AND INDUCES REGRESSION OF ESTABLISHED EXPERIMENTAL DERMAL FIBROSIS INDUCED BY BLEOMYCIN}

H. Štorkánová ${ }^{1,2}$, L. Štorkánová ${ }^{1}$, S. Oreska ${ }^{1,2}$, M. Špiritovici ${ }^{3}$, B. Heřmánková ${ }^{3}$, R. Bečváŕr, ${ }^{1,2}$, K. Pavelka ${ }^{1,2}$, J. Vencovský ${ }^{1,2}$, J. Distler ${ }^{4}$, L. Šenolt ${ }^{1,2}$ M. Tomcik ${ }^{1,2} .{ }^{1}$ Institute of Rheumatology, Prague, Czech Republic; ${ }^{2}$ Department of Rheumatology, 1st Faculty of Medicine, Charles University, Prague, Czech Republic, Prague, Czech Republic; ${ }^{3}$ Faculty of Physical Education and Sport, Charles University, Prague, Czech Republic, Prague, Czech Republic; ${ }^{4}$ Department of Internal Medicine III and Institute for Clinical Immunology, University of Erlangen-Nuremberg, Erlangen, Germany, Erlangen, Germany

Background: Our previous study demonstrated that Heat shock protein 90 (Hsp90) is overexpressed in the skin of patients with systemic sclerosis (SSc), in cultured SSc fibroblasts and preclinical models of SSc. HSP90 is a new regulator of canonical TGF- $\beta$ signalling and its inhibition prevents the stimulatory effects of TGF- $\beta$ on collagen synthesis and dermal fibrosis in three preclinical models of SSc.

Objectives: Herein, we aimed to evaluate the efficacy of Hsp90 inhibitor (17DMAG) in the treatment of established experimental dermal fibrosis induced by bleomycin.

Methods: Design consisted of three control groups, I ( $\mathrm{NaCl}$-s.c./6 weeks), II (bleomycin-s.c./3w and $\mathrm{NaCl}$-s.c./3w), III (bleomycin-s.c./6w), and 2 treatment groups (bleomycin-s.c./6w). During the last 3 weeks, one group was treated with 17-DMAG $0.5 \mathrm{mg} / \mathrm{kg}$-i.p. every third day, whereas one group (with nintedanib $50 \mathrm{mg} / \mathrm{kg}$-p.o. twice daily) served as a comparator with already published efficacy in this setting. Total of 40 BL6 mice were examined weekly for weight, activity and fur texture. The effects of 17-DMAG were determined by assessment of dermal thickness (HE-staining), collagen content (hydroxyproline assay), myofibroblast 